\title{
A new angiographic finding: primary peripheral slow flow
}

\author{
Ercan Akşit ${ }^{1} \oplus$, Emine Gazi ${ }^{1} \odot$, Bahadır Kırılmaz ${ }^{1} \oplus$, Fatih Aydın² \\ ${ }^{1}$ Department of Cardiology, Çanakkale Onsekiz Mart University School of Medicine, Çanakkale, Turkey \\ ${ }^{2}$ Clinic of Cardiology, Eskisehir City Hospital, Eskişehir, Turkey
}

To the Editor,

In some peripheral angiographies, similar to the coronary slow flow phenomenon (CSFP), we observed a peripheral slow flow (Supplementary Video 1). Although slow flow phenomenon after infrapopliteal balloon angioplasty is mentioned in 2 retrospective studies $^{1,2}$, primary peripheral slow flow has been previously reported in only 1 case report. ${ }^{3}$ Interestingly, it appears that similar to CSFP sometimes occurring in a single coronary artery, there is slow flow only in the left lower extremity artery in the video example (Supplementary Video 1). There is also no severe stenosis that would explain this phenomenon. Moreover, considering that peripheral angiography was performed with the right femoral artery puncture and that the catheter was within the right femoral artery, it could have been expected for the flow to be slower in the right lower extremity artery with the effect of spasm. However, in this video example, it appears that the flow was slower on the left side, where there was no catheter. Researching the etiologies that can cause peripheral slow flow can allow us to reach new horizons in both the diagnosis and treatment of peripheral vascular diseases. Possible reasons that may cause peripheral slow flow are presented in Table 1. One of the possible etiologies that deserve to be researched is May-Thurner syndrome. ${ }^{4}$ This syndrome is a clinical condition that can occur with iliofemoral thrombosis due to the right common iliac artery compressing the left common iliac vein on the lumbar vertebra. The compression effect in this anatomical variation can be one of the secondary causes of peripheral slow flow. When patients describe claudication, they usually undergo peripheral arterial angiography, and the observation of peripheral slow flow in these patients may spring to mind the under-recognized May-Thurner syndrome (claudication is observed prevalently also among these patients) for differential diagnosis.

The researchers noted that when the infrapopliteal cine frame count (CFC) is $>35$ (between infrapopliteal artery and ankle joint), it is considered an infrapopliteal slow flow after an endovascular intervention. Slow flow after the procedure has been associated with worse wound healing. ${ }^{1,2}$ In our example, CFC was 297 for the left lower extremity and 129 for the right lower extremity (at 15 frames/s) (between external iliac artery and ankle joint). In this patient, peripheral angiography of both lower extremities was performed by the right femoral artery approach using a $6 \mathrm{~F}$ pigtail catheter with the same protocol $(15 / 35=$ by injecting $15 \mathrm{~mL}$ contrast per second, $35 \mathrm{~mL}$ contrast in total) and the same automated injection device (Liebel-Flarsheim Company, Cincinnati, OH, USA). The CFC used to detect slow flow in peripheral angiography is more objective than the TIMI frame count $^{5}$ used to detect CSFP. Because coronary angiography is performed manually, the flow speed of the opaque medium in the coronary artery varies from operator to operator. Conversely, peripheral angiographies are performed with an automated injection device under certain standards and protocol, and this provides a more objective evaluation of peripheral slow flow with the CFC method.

Because cardiovascular diseases are the leading cause of mortality and morbidity, research is ongoing to understand these diseases. ${ }^{6}$ As a new definition, primary peripheral slow flow phenomenon may explain claudication complaints without any other underlying causes when it is detected in the extremity arteries and may explain neurological symptoms without any other underlying causes when it is detected in carotid or vertebral arteries. Having observed both coronary and peripheral slow flow in some patients brings forth the

TABLE 1. Possible Causes of Peripheral Slow Flow Phenomenon

\begin{tabular}{ll}
\hline Primary causes & Secondary causes \\
\hline $\begin{array}{l}\text { It is associated with local or systemic (to- } \\
\text { gether with or without coronary slow flow) }\end{array}$ & Peripheral arterial stenosis \\
endothelial dysfunction or microvascular & Peripheral arterial embolism \\
disease. & Peripheral arterial spasm \\
& Peripheral arterial ectasia \\
& Connective tissue disorders \\
& External compression effect \\
& (e.g., May-Thurner syndrome) \\
& Peripheral arterial interventions \\
\hline
\end{tabular}

Address for Correspondence: Ercan Akșit, Department of Cardiology, Canakkale Onsekiz Mart University School of Medicine, Canakkale, Turkey e-mail: ercanaksit@comu.edu.tr

Received: July 2, 2020 Accepted: September 15, 2020 •DOI: 10.4274/balkanmedj.galenos.2020.2020.7.30

Available at www.balkanmedicaljournal.org

ORCID iDs of the authors: E.A. 0000-0002-4478-4324; E.G. 0000-0002-0513-0870; B.K. 0000-0002-5929-1426; F.A. 0000-0002-1017-1917.

Cite this article as:

Akşit E, Gazi E, Kırılmaz B, Aydın F. A new angiographic finding: primary peripheral slow flow. Balkan Med J. 2021;38(2):139-140.

Copyright@Author(s) - Available online at http://balkanmedicaljournal.org/ 
idea that primary slow flow phenomenon may be due to a systemic endothelial dysfunction or microvascular disease in some patients. Because gene mutations are shown in both peripheral slow flow and coronary slow flow, ${ }^{3,7}$ recognizing systemic slow flow may lead to genetic studies that will try to reveal new causes of thrombosis predisposition.

Patient Consent for Publication: Written and verbal consent was obtained from the patient.

Author contributions: Concept - E.A.; Design - E.A., E.G., B.K.; Supervision - B.K.; Resources - E.A., E.G.; Materials - E.G., B.K.; Analysis and/or Interpretation - F.A.; Literature Review - E.G., F.A.; Writing - E.A., F.A.; Critical Review - B.K., F.A.

Conflict of Interest: The authors have no conflicts of interest to declare.

Funding: The authors declared that this study has received no financial support.

SUPPLEMENTARY VIDEO 1. There is slow flow only in the left lower extremity artery in the video example. Cine frame count was 297 for the left lower extremity and 129 for the right lower extremity (at 15 frames/s) (between external iliac artery to ankle joint).

\section{REFERENCES}

1. Kobayashi N, Hirano K, Yamawaki M, et al. Clinical impact and predictors of the slow-flow phenomenon after endovascular treatment of infrapopliteal lesions using the crosser catheter in patients with critical limb ischemia. $J$ Vasc Interv Radiol. 2020;31(1):141-149. [Crossref]

2. Tokuda T, Hirano K, Sakamoto Y, et al. Incidence and clinical outcomes of the slow-flow phenomenon after infrapopliteal balloon angioplasty. J Vasc Surg. 2017;65(4):1047-1054. [Crossref]

3. Akşit E, Gazi E, Toprak CA, et al. Unilateral peripheral slow flow phenomenon without significant stenosis in lower extremity artery: can primary peripheral slow flow be a new phenomenon? BMJ Case Rep. 2020;13(9):e235686. [Crossref]

4. May R, Thurner J. The cause of the predominantly sinistral occurrence of thrombosis of the pelvic veins. Angiology. 1957;8(5):419-427. [Crossref]

5. Gibson CM, Cannon CP, Daley WL, et al. TIMI frame count: a quantitative method of assessing coronary artery flow. Circulation. 1996;93(5):879-888. [Crossref]

6. Çakmak HA, Demir M. MicroRNA and cardiovascular diseases. Balkan Med J. 2020;37(2):60-71. [Crossref]

7. Tang $\mathrm{O}, \mathrm{Wu}$ J, Qin F. Relationship between methylenetetrahydrofolate reductase gene polymorphism and the coronary slow flow phenomenon. Coron Artery Dis. 2014;25(8):653-657. [Crossref] 